\title{
Increased sural nerve epineurial blood flow in human subjects with painful diabetic neuropathy
}

\author{
S. E. M. Eaton 1 , N. D. Harris ${ }^{2}$, S. Ibrahim 1 , K. A. Patel ${ }^{3}$, F. Selmi ${ }^{4}$, M. Radatz ${ }^{4}$, J. D. Ward ${ }^{1}$, S. Tesfaye ${ }^{1}$ \\ ${ }^{1}$ Diabetes Research Unit, Royal Hallamshire Hospital, Sheffield, UK \\ 2 Department of Medical Physics, Royal Hallamshire Hospital, Sheffield, UK \\ ${ }^{3}$ Department of Surgical and Anaesthetic Sciences, Royal Hallamshire Hospital, Sheffield, UK \\ ${ }^{4}$ Department of Neurosurgery, Royal Hallamshire Hospital, Sheffield, UK
}

\begin{abstract}
Aims/hypothesis. The pathogenesis of painful diabetic neuropathy remains unknown. As a consequence we still do not have any effective, rational treatments and a greater understanding of the mechanisms is urgently required. Previous studies have shown no consistent morphological differences in the nerves of patients with and without painful neuropathy. The aim of this study was to compare epineurial haemodynamics in patients with chronic painful and painless neuropathy.

Methods. The techniques of microlightguide spectrophotometry and fluorescein angiography were used to measure epineurial intravascular oxygen saturation and blood flow respectively. Eleven patients with painful and eight with painless neuropathy were studied, with the groups matched carefully in terms of severity of neuropathy and diabetes control.
\end{abstract}

Results. Intravascular oxygen saturation was higher in the painful neuropathy group compared to those without pain (median $73.8 \%$ vs $67.7 \%$, respectively; $p=0.021$ ). Fluorescein rise time was also faster in those with painful symptoms (median $18.3 \mathrm{~s}$ vs $53.6 \mathrm{~s}$; $p=0.046$ ) indicating higher epineurial blood flow in these subjects.

Conclusion/interpretation. These results indicate that there are distinct differences in haemodynamics within the epineurium of the sural nerve in subjects with painful and painless neuropathy. Haemodynamic factors could therefore have an important role in the pathogenesis of neuropathic pain and might offer further insight into potential treatments for this distressing condition. [Diabetologia (2003) 46:934-939]

Keywords Diabetes mellitus, diabetic neuropathy, painful, pain, hypoxia, epineurium, fluorescein angiography, spectrophotometry.
Distal symmetrical polyneuropathy (DSP) is a common chronic complication of diabetes mellitus [1]. Despite extensive research, its pathogenesis remains unclear, although a variety of vascular and metabolic factors have been implicated [2]. A proportion of pa-

Received: 21 November 2002 / Revised: 18 March 2003 Published online: 19 June 2003

C) Springer-Verlag 2003

Corresponding author: Dr. S. Tesfaye, Diabetes Research Unit, Royal Hallamshire Hospital, Glossop Road, Sheffield, UK E-mail: solomon.tesfaye@sth.nhs.uk

Abbreviations: DSP, distal symmetrical polyneuropathy; FRT, fluorescein rise time; $\mathrm{HbO}_{2} \%$, intravascular oxygen saturation; $\mathrm{NCV}$, nerve conduction velocity; VPT, vibration perception threshold. tients with DSP suffer painful neuropathic symptoms that can be very distressing, often disturbing the person's ability to sleep or function normally [3]. Neuropathic pain arises in the absence of nocioceptive stimuli and serves no apparent biological purpose [4]. Current treatments are unsatisfactory, as they are not based on a robust understanding of the mechanisms of the pain and are frequently ineffective, often with intolerable side-effects $[5,6]$.

The cause of painful DSP is unclear and there is no good explanation as to why some people with DSP suffer pain and others do not. Nerve morphology has been investigated extensively but no consistent differences to explain the pain have been found $[7,8,9,10$, $11,12]$. Similarly, comparisons of structural microvascular disease in painful and painless DSP have been 
inconsistent. Another study found a reduction in capillary basement membrane thickening in painful neuropathy compared to subjects with recurrent foot ulceration [13] however, these differences have not been confirmed in other studies using more comparable subjects [10].

Impaired nerve blood flow and hypoxia are known to be important factors in the pathogenesis of DSP $[14,15]$, although they have not previously been compared in subjects with chronic painful and painless DSP. However, there is reason to suspect that vascular factors could be important in pain. The fluctuant nature of painful neuropathic symptoms might suggest a more dynamic underlying cause, such as metabolic or haemodynamic factors, rather than structural lesions. Furthermore, it is recognized that manoeuvres that reduce lower limb blood flow, including vessel occlusion and cooling of the limb, can reduce painful neuropathic symptoms [16]. The aim of this study was to compare sural nerve epineurial haemodynamics in subjects with chronic painful and painless DSP.

\section{Subjects and methods}

We studied 11 subjects with chronic painful DSP and eight subjects with chronic painless DSP. The presence of DSP was defined according to detailed neurological examination, quantitative sensory testing and nerve conduction testing [17]. Subjects were considered to have painful DSP if they described the typical "positive" symptoms (including paraesthesia, burning or shooting pains) which were persistent ( $>1$ year), required treatment and had disturbed sleep, work or recreational activities.

All subjects underwent the following: (i) full history and examination including neuropathic symptoms and deficit scores [17] (ii) peroneal motor and sensory nerve conduction, measured at a skin temperature of $32 \pm 1^{\circ} \mathrm{C}$ at a room temperature of $24 \pm 2^{\circ} \mathrm{C}$, using a Dantec $2000 \mathrm{M}$ electrophysiological system with signal averaging (Dantec, Bristol, UK), (iii) vibration perception threshold at the great toe using the biothesiometer (Biomedical Instrument, Newbury, Ohio, USA), (iv) cardiac autonomic function tests, carried out with a computer assisted technique according to a defined protocol [18], and (v) ankle brachial pressure index using a Doppler ultrasound stethoscope, model BF4A (Med. Sonics, Mountain View, Calif., USA).

Subjects were excluded from the study if they had any of the following: (i) peripheral vascular disease $(>1$ absent foot pulse or an ankle-brachial pressure index $<0.7$ ), (ii) were current smokers, (iii) were taking vasoactive drugs (including antihypertensives), or (iv) neuropathy from causes other than diabetes mellitus.

All subjects gave their informed consent and the local ethics committee approved the study.

The equipment and methods used in the measurement of epineurial intravascular oxygen saturation and blood flow have been validated and described in detail elsewhere [15, 19].

Sural nerve exploration. The position of the sural nerve was mapped out using small bipolar electrical stimulating electrodes, identifying points of maximal sensitivity along the presumed course of the nerve. The subject was then made com- fortable on the operating table in a daycase theatre and the foot immobilised in a cradle. The skin was infiltrated with $2 \%$ plain Lidocaine and a short segment $(1 \mathrm{~cm})$ of the sural nerve was exposed through a skin incision and blunt dissection by an experienced surgeon. As in our previous studies, great care was taken to avoid disturbing the nerve and its vasculature. A needle thermocouple was placed alongside the nerve to monitor temperature throughout the procedure [15].

Intravascular oxygen saturation measurement $\left(\mathrm{HbO}_{2} \%\right)$. Measurements were done using the Erlangen microlightguide spectrophotometer II (EMPHO II, Bodenseewerk Gerätetechnik, Überlingen, Germany) [15, 19]. Light was shone onto the surface of the nerve through an endoscope and reflected light was collected, fed through a rotating interference filter disc and monochromatised in a range between 502 to $628 \mathrm{~nm}$. The shape of the measured haemoglobin spectra was compared to reference spectra and $\mathrm{HbO}_{2} \%$ was calculated by an on-line program [20].

We took three sets of $100 \mathrm{HbO}_{2} \%$ measurements at different sites along the nerve and these were averaged. The endoscope was mounted on a micromanipulator and guided into position, $0.5 \mathrm{~mm}$ from the surface of the nerve, using the image of the nerve shown on a video screen. After each set of measurements the tip of the endoscope and the exposed nerve were washed with warm saline and dried.

Measurement of fluorescein rise time (FRT). Each subject was given a bolus injection of $20 \%$ Sodium Fluorescein (Martindale Pharmaceuticals, Romford, UK) into the right cephalic vein at a dose of 1 millilitre per $20 \mathrm{~kg}$ body weight $[15,19]$. Fluorescein excitation was elicited using a $250 \mathrm{~W}$ Halogen light source with a $485 \mathrm{~nm}$ blue filter. Nerve fluorescence was measured with the endoscope tip $1 \mathrm{~mm}$ away from the surface of the nerve for a period of $5 \mathrm{~min}$. The position of the endoscope and nerve was monitored and recorded on video. Subjects in whom there was any appreciable change in the position of the nerve under the endoscope were excluded.

Nerve fluorescence was quantified directly from the uncorrected spectra by subtracting the baseline intensity values and integrating between 510 and $560 \mathrm{~nm}$. The time between $10 \%$ and $90 \%$ maximum intensity was calculated to determine the fluorescein rise time (FRT), a measure of epineurial blood flow [15, 21].

Statistics. Statistical analysis was carried out using SPSS version 9.0. The $\mathrm{HbO}_{2} \%$ and FRT data was analyzed using Mann-Whitney U test. Linear correlation was analyzed using Pearson's Coefficient. A $p$ value of less than 0.05 was considered statistically significant.

\section{Results}

The painless DSP group were slightly older than those with pain but this difference was not statistically significant. There was no difference in duration and control of diabetes between the two groups. Both groups were well matched in terms of neurophysiological parameters, including sural and peroneal NCV, VPT and cardiac autonomic function tests (Table 1).

Intravascular oxygen saturation measurement $\left(\mathrm{HbO}_{2} \%\right)$. Oxygen saturation was higher in those subjects with painful symptoms compared to those without pain 


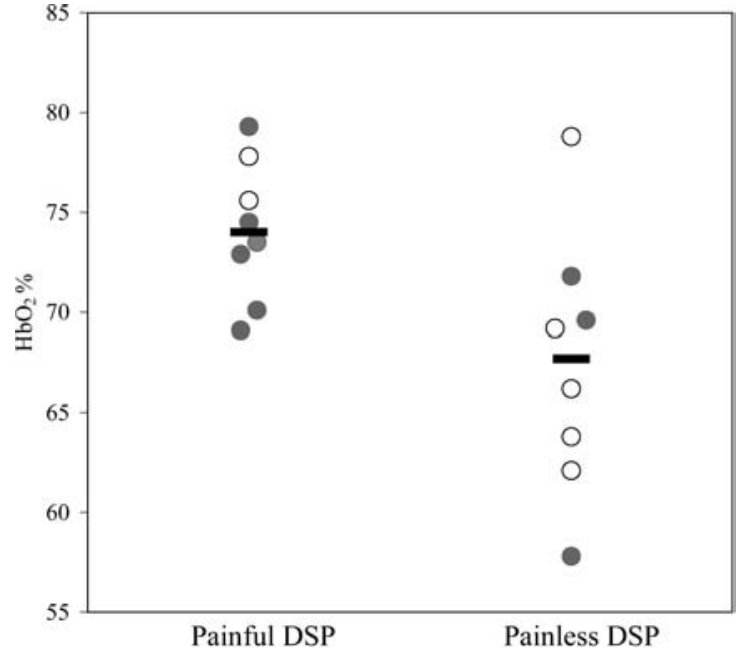

Fig. 1. Sural nerve intravascular oxygen saturation $\left(\mathrm{HbO}_{2} \%\right)$ in subjects with painful and painless DSP $(p=0.021$, MannWhitney U test). Bars represent median values. Empty circles represent patients with T1DM, filled circles T2DM

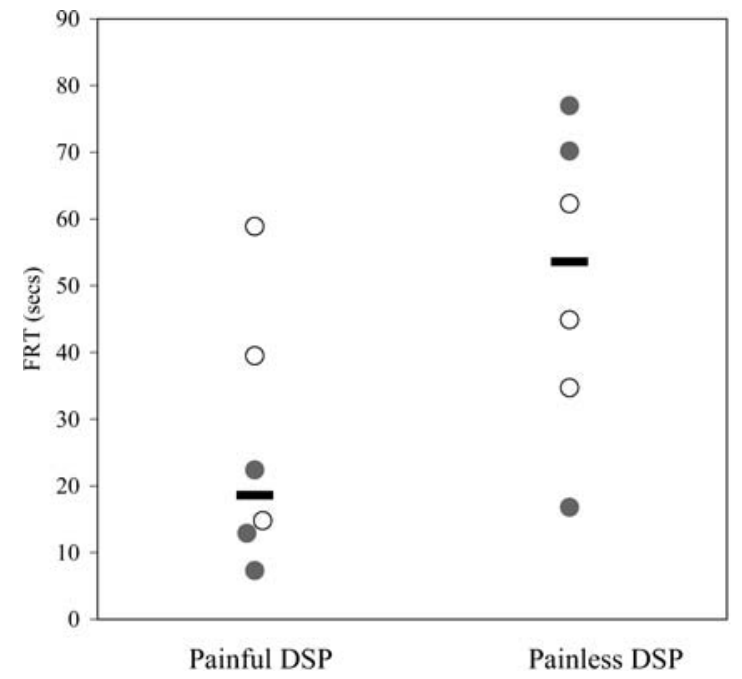

Fig. 2. Fluorescein rise time (FRT) in subjects with painful and painless DSP ( $p=0.046$, Mann-Whitney U Test). Bars represent median values. Empty circles represent patients with T1DM, filled circles T2DM

Table 1. Clinical characteristics of study subjects

\begin{tabular}{lcr}
\hline GROUPS & Painless DSP $(n=8)$ & Painful DSP $(n=11)$ \\
\hline Age (years) & $57.5(11.0)$ & $51.0(10.1)$ \\
Sex Male/Female & $7: 1$ & $9: 2$ \\
DM Type 1/2 & $5: 3$ & $4: 7$ \\
Duration (years) & $15.4(10.8)$ & $15.4(9.4)$ \\
Glycated haemoglobin (\%) & $8.4(2.7)$ & $9.1(3.1)$ \\
Sural Sensory NCV (m/s) & $28.4(7.3)$ & $33.5(9.8)$ \\
Peroneal Motor NCV (m/s) & $31.8(6.5)$ & $31.0(7.2)$ \\
VPT (volts) & $33.8(17.9)$ & $33.8(15.3)$ \\
One or more abnormal cardiac autonomic function tests $(\%)$ & 38 & 45
\end{tabular}

Expressed as means (SD) unless otherwise stated

Table 2. Correlations between sural nerve oxygen saturation $\left(\mathrm{HbO}_{2} \%\right)$ and fluorescein rise time (FRT) and other demographic and neurophysiological parameters

\begin{tabular}{|c|c|c|c|c|}
\hline \multirow[b]{2}{*}{ Variable } & \multicolumn{2}{|c|}{ Correlation with $\mathrm{HbO}_{2} \%$} & \multicolumn{2}{|l|}{ Correlation with FRT } \\
\hline & Pearson correlation & Significance $p$ & Pearson correlation & Significance $p$ \\
\hline Age & -0.152 & 0.559 & 0.223 & 0.464 \\
\hline Glycated haemoglobin & -0.064 & 0.807 & 0.437 & 0.136 \\
\hline Sural Sensory NCV & 0.039 & 0.881 & -0.311 & 0.301 \\
\hline Expiration/Inspiration $\mathrm{R}$ to $\mathrm{R}$ ratio & 0.127 & 0.728 & -0.696 & 0.125 \\
\hline
\end{tabular}

(Statistically significant correlations shown in bold type)

(median $73.8 \%$ vs $67.7 \%, p=0.021$ ) (Fig. 1). Measurements of $\mathrm{HbO}_{2} \%$ were carried out at three or more positions along the nerve. The coefficient of variation of these measurements was $5.02 \%$.

Fluorescein rise time (FRT). FRT was faster in the painful DSP group (median $18.3 \mathrm{~s}$ vs $53.6 \mathrm{~s}, p=0.046$ ) compared to those without pain, indicating higher blood flow in those subjects with pain (Fig. 2).

Correlation between $\mathrm{HbO}_{2} \%, \mathrm{FRT}$ and other neurophysiological and demographic parameters. As in previous studies, there was a correlation between FRT and $\mathrm{HbO}_{2} \%$, confirming that the changes in oxygen 
status are due to differences in nerve blood flow $(\mathrm{r}=-0.711, p=0.014)$ (Table 2). There was no correlation between either FRT or $\mathrm{HbO}_{2} \%$ with age, duration of diabetes or glycaemic control. Furthermore, neither parameter correlated with any of the measured neurophysiological parameters, including sural and peroneal NCV, VPT or cardiac autonomic function tests. There was no influence of the type of diabetes on the results for either $\mathrm{HbO}_{2} \%$ or FRT.

\section{Discussion}

Painful neuropathy is a common problem in the care of diabetic patients. The sad reality, however, is that the pathogenesis is poorly understood and we are frequently unable to provide effective, tolerable treatments. Available treatments include tricyclic anti-depressants, anticonvulsants, opiate analgaesia, topical capsaicin and lidocaine infusions [5, 22]. Despite some evidence of proven benefit for each of these agents, they are often ineffective and are frequently associated with unacceptable side effects at therapeutic dosages. Furthermore, each of these treatments are aimed at symptom control and do not target the underlying mechanisms generating the pain. A greater understanding of the causes of painful symptoms of neuropathy is crucial to the development of newer, rational therapeutic agents.

We have shown that there are striking differences in sural nerve haemodynamics in subjects with painful and painless DSP. Epineurial $\mathrm{HbO}_{2} \%$, measured using microlightguide spectrophotometry, was higher in the group with painful neuropathic symptoms compared to those without. Furthermore, epineurial blood flow, as measured using FRT, was faster in those with pain. The correlation between $\mathrm{HbO}_{2} \%$ and FRT indicates that the changes in oxygen status are mainly due to differences in blood flow rather than tissue oxygen extraction.

These results indicate that haemodynamic differences exist within the nerve in painful and painless neuropathy. Before we can consider what these differences mean to the mechanisms of painful DSP we need to establish exactly what we are measuring and what these differences reflect. Painful neuropathic symptoms are frequently independent of the severity of neuropathy and do occasionally occur with near-normal nerve function [12]. Previous work has shown that structural microvascular disease is strongly correlated with neurophysiological parameters [23] and there are no differences in those with painful and painless DSP [10]. Thus, differences in the severity of neuropathy in patients with and without painful symptoms have the potential to bias any comparative work. For this reason, we have taken great care to ensure both groups were matched accurately in this study, particularly in terms of neurophysiological parameters.
Microlightguide spectrophotometry and fluorescein angiography were developed to replace an earlier technique of microelectrode polarography, which required repeated insertion of a microelectrode into the nerve to measure endoneurial oxygen saturation [24]. The resultant nerve trauma has meant that this technique is no longer felt appropriate in studies of human subjects, although it is still widely and successfully used in experimental animal studies. The newer techniques are non-invasive to the nerve and, whilst this results in an absence of trauma, it does have the limitation of predominantly focusing on the epineurium. It is important to be clear about this when interpreting the results of our study and considering the relevance to the cause of pain. We have shown differences between the two groups in epineurial blood flow and oxygenation. We cannot conclude with any certainty what is happening to these parameters within the endoneurium. However, currently we have no acceptable means of directly measuring this area and must therefore speculate from the evidence that is available. Morphometric studies in DSP have shown that structural microvascular disease is present throughout the nerve, but is more marked in the endoneurium than the epineurium [25]. Groups comparing those with and without pain have shown no consistent morphometric differences within the endoneurium [10] but the epineurium has not been studied in this context. There has never been any direct comparison of the techniques assessing endoneurial and epineurial oxygen saturations, although both areas show very similar reductions in patients with DSP $[15,24]$. Thus, we know there is endoneurial hypoxia in DSP, and marked endoneurial microangiopathy is present in patients with pain, similar to those without pain. We would therefore suggest that, although we have shown increased epineurial oxygen saturation and blood flow in pain, the endoneurium is likely to still be hypoxic. As we have stated, however, there is no acceptable method available to clarify this situation.

In this study, we have observed dramatically higher blood flow and oxygen saturation in the epineurium of those subjects with painful DSP compared to those without pain. Intriguingly, the results in the painful group were remarkably similar to the values previously measured, using an identical techniques and protocol, in non-neuropathic diabetic subjects and normal control subjects [15]. Yet, these would be subjects in whom we would expect minimal or no microvascular disease [23]. Furthermore, in this study, subjects did have DSP and we were extremely careful to match the groups in terms of severity, to ensure that structural microvascular disease is likely to be comparable. Therefore, these results suggest that there is an extraordinary increase in blood flow within the epineurium of subjects with painful DSP, which occurs despite the presence of microvascular disease. 
The factors that influence blood flow in painful DSP, and the relevance these have on the mechanisms of pain, remain unclear. Our group has previously described arteriovenous shunting associated with neovascularisation on the nerve surface in subjects with acute painful neuropathy [26]. At that time we hypothesized that the arteriovenous shunting led to a "steal effect" rendering the endoneurium profoundly hypoxic, which could have been the stimulus for pain. The results of this study suggest a similar phenomenon is occurring in chronic painful DSP. Blood shunted from the endoneurium would produce an increase of blood flow in the epineurium, causing higher oxygen saturations. There are a number of potential causes of arteriovenous shunting including local sympathetic denervation [27]. We have made crude measurements of cardiac autonomic function in this study and these were similar in both groups of subjects, but this might not accurately reflect autonomic integrity at the level of the nerve. Other causes of arteriovenous shunting include the release of vasoactive neurotransmitters involved in the generation or mediation of pain. Indeed, increased levels of noradrenaline have been observed in the blood of patients with PDN and correlated with the severity of pain [28]. Further work assessing differences in local autonomic responses and neurotransmitters in painful DSP is required.

Further evidence to support the assertion that increased blood flow is relevant to the cause of pain is gained from the observation that painful symptoms might improve with maneouvres that reduce blood flow to the limb [16]. In the process of studying blood flow in neuropathy, it was observed that, on inflation of a sphygmomanometer cuff to measure blood flow in the limb, subjects with painful neuropathy often reported that symptoms improved in that limb. This has additional implications to the treatment of painful symptoms and suggests that therapies that reduce blood flow and/or shunting could prove to be of benefit in reducing pain.

In summary, we have shown a distinct physiological difference within the nerve in subjects with and without painful symptoms of DSP. Epineurial blood flow and oxygen saturation are increased in those with pain, possibly as a consequence to arteriovenous shunting. This in turn could be exacerbating endoneurial hypoxia, which might be important in the generation of the pain. Further work is required to clarify the nature of these differences and their relevance to the mechanisms of pain. This work could lead to a more thorough understanding of pain in DSP and potentially, to the development of more rational and effective treatments.

\section{References}

1. Eaton SEM, Tesfaye S (1999) Clinical manifestations and measurement of somatic neuropathy. Diabetes Rev 7:312325

2. Cameron NE, Eaton SEM, Cotter MA, Tesfaye S (2001) Vascular factors and metabolic interactions in the pathogenesis of diabetic neuropathy. Diabetologia 44:1973-1988

3. Watkins PJ (1984) Pain and diabetic neuropathy. BMJ 228:168-169

4. Woolf C, Mannion R (1999) Neuropathic pain: aetiology, symptoms, mechanisms and management. Lancet 353: 1959-1964

5. Young RJ (1997) The management of diabetic neuropathy. In: Pickup J, Williams G (eds) Textbook of diabetes, 2nd edn. Blackwell, Oxford, pp 51.1-51.16

6. Tesfaye S, Watt J, Benbow SJ, Pang KA, Miles J, MacFarlane IA (1996) Electrical spinal-cord stimulation for painful diabetic peripheral neuropathy. Lancet 348: 1698-1701

7. Asbury AK, Fields HL (1984) Pain due to peripheral nerve damage; an hypothesis. Neurology 34:1587-1590

8. Britland ST, Young RJ, Sharma AK, Clarke BF (1990) Association of painful and painless diabetic polyneuropathy with different patterns of nerve fiber degeneration and regeneration. Diabetes 39:898-908

9. Llewelyn JG, Gilbey SG, Thomas PK, King RHM, Muddle JR, Watkins PJ (1991) Sural nerve morphometry in diabetic autonomic and painful sensory neuropathy. Brain 114:867892

10. Malik R (1997) The pathology of human diabetic neuropathy. Diabetes 46 [Suppl 2]:S50-S53

11. Brown MJ, Martin JR, Asbury AK (1976) Painful diabetic neuropathy: a morphometric study. Arch Neurol 33:164171

12. Young RJ, Zhou YQ, Rodriguez E, Prescott RJ, Ewing DJ, Clarke BF (1986) Variable relationship between peripheral somatic and autonomic neuropathy in patients with different syndromes of diabetic polyneuropathy. Diabetes 35: 192-197

13. Britland ST, Young RJ, Sharma AK, Clarke BF (1990) Relationship of endoneurial capillary abnormalities to type and severity of diabetic neuropathy. Diabetes 39:909-913

14. Low PA (1987) Recent advances in the pathogenesis of diabetic neuropathy. Muscle Nerve 10:121-128

15. Ibrahim S, Harris N, Radatz M et al. (1999) A new minimally invasive technique to show nerve ischaemia in diabetic neuropathy. Diabetologia 42:737-742

16. Archer AG, Roberts VC, Watkins PJ (1984) Blood flow patterns in painful diabetic neuropathy. Diabetologia 27:563-567

17. Dyck PJ (1988) Detection, characterization and staging of polyneuropathy: assessed in diabetics. Muscle Nerve 11: $21-32$

18. O'Brien IAH, O'Hare P, Corrall RJM (1986) Heart rate variability in healthy subjects: effect of age and derivation of normal ranges for tests of autonomic function. Br Heart J 55:348-354

19. Ibrahim S, Laude E, Bee D, Eaton S, Ward JD, Harris ND (1999) Combined microlightguide spectrophotometry and microendoscopy for measurement of oxygen saturation in peripheral nerves. Physiol Meas 20:65-73

20. Frank K, Kessler M, Appelbaum K, Dummler W (1989) The Erlangen micro-lightguide Spectrophotometer EMPHO I. Phys Med Biol 34:1883-1900

21. Perbeck L, Lund F, Svensson L, Thulin L (1985) Fluorescein flowmetry: a method for measuring relative capillary blood flow in the intestine. Clin Physiol 5:281-292 
22. Tesfaye S, Price DE (1997) Therapeutic approaches in neuropathy and neuropathic pain. In: Boulton AJM (ed.) Diabetic neuropathy, 1st edn. Marius Press, Lancashire, pp 159-181

23. Malik R, Newrick PG, Sharma AK et al. (1989) Microangiopathy in human diabetic neuropathy; relationship between capillary abnormalities and the severity of neuropathy. Diabetologia 32:92-102

24. Newrick PG, Wilson AJ, Jakubowski J, Boulton AJM, Ward JD (1986) Sural nerve oxygen tension in diabetes. BMJ 293:1053-1054
25. Malik R, Tesfaye S, Thompson SD et al. (1993) Endoneurial localisation of microvascular damage in human diabetic neuropathy. Diabetologia 36:454-459

26. Tesfaye S, Malik R, Harris ND et al. (1996) Arterio-venous shunting and proliferating new vessels in acute painful neuropathy of rapid glycaemic control (insulin neuritis). Diabetologia 39:329-335

27. Watkins PJ, Edmonds ME (1983) Sympathetic nerve failure in diabetes. Diabetologia 25:73-77

28. Tsigos C, Reed P, Weinkove C, White A, Young RJ (1993) Plasma norepinepherine in sensory diabetic polyneuropathy. Diabetes Care 16:722-727 